Magnetic Separation News, 1986, Vol. 2, pp. 91-94

0731-3632/86/0202-0091 \$25.00/0

(1) 1986 Gordon and Breach, Science Publishers, Inc.

Printed in the United Kingdom

\title{
A PRECAUTION ON USING 316L STAINLESS WIRE IN HGMS MATRICES *
}

\author{
MARSHALL D. GRAHAM \\ Coulter Biomedical Research Corporation, 35 Wiggins Avenue, \\ Bedford, Massachusetts 01730 , U.S.A.
}

\begin{abstract}
Use of unannealed 316L stainless wire as HGMS matrix material in an application requiring frequent reversals of the magnetizing field has been noted to result in slow degradation of matrix magnetization characteristics, with attendant reductions in magnetic filtration efficiency. This alloy should be restricted to applications where field reversals are seldom required, particularly if it is exposed to corroding fluids. A similar precaution probably applies to other austenitic alloys.
\end{abstract}

\section{INTRODUCTION}

Austenitic stainless alloys are sometimes used in the work-hardened state as a matrix material in HGMS systems ${ }^{1-3}$. In applications requiring exposure to saline, their superior corrosion resistance may offset their comparatively inferior magnetic properties. Occasionally, the latter also offer experimental advantages.

During work with an experimental method for magnetic filtration of blood cells ${ }^{4}$, calculations had predicted that cellular magnetic contrasts would be several times greater than with usual methods ${ }^{5}$. It thus seemed logical to use work-hardened, 316L austenitic stainless wire as matrix, since its lower saturation magnetization would give a more strenuous test of the new method than were 430 ferritic stainless wire used. The expected filtration efficiency was initially attained, and run-to-run repeatability was excellent ${ }^{4}$. However the protocol required elutriation of the retained cells by reducing the magnetizing field to as nearly zero as possible. This was done by alternating the magnetization direction at decreasing field magnitudes while monitoring the remanent magnetization of the HGMS system. After several experimental runs it was noted that filtration efficiencies had begun to decrease, with attendant increase in variability between consecutive filtration runs. Considerable effort was spent tracing the variability to the 316L matrix, which behaved magnetically as though it were being progressively annealed.

* Presented at the 16th Annual Meeting of the Fine Particle Society, 22-25 April 1985, Miami Beach, Florida. 
METHODS

Detailed descriptions of the experimental protocol are available 4 , as is one of the filter ${ }^{6}$. Unannealed $316 \mathrm{~L}$ wire 35 micra in diameter was used to pack the filter chamber to $11.4 \%$ of its volume. Diluted 1:101 with an experimental reagent, whole human blood was pumped at $0.75 \mathrm{ml} / \mathrm{min}$ through the filter with the $10 \mathrm{~cm}$ electromagnet energized to provide the desired magnetization field. After passage of $1.2 \mathrm{ml}$ of sample the pump was stopped, and remanent magnetization measured at the filter was reduced to less than a mTesla, by reversing connections of the electromagnet to its power supply several times and re-energizing the magnet at progressively decreasing field strengths. The captured cells were then elutriated by flushing the filter with either the experimental diluent or, in the case of dithionite controls, isotonic saline $(0.9 \% \mathrm{NaCl})$. The experimental diluents contained $\mathrm{ErCl}_{3}$ at various concentrations, and ones below $35 \mathrm{mM}$ content had osmolarities adjusted to $300 \mathrm{mOsmol}$ by addition of $3 \mathrm{M} \mathrm{NaCl}$.

Filtration efficiencies were calculated as in Reference 4. On realization that filtration characteristics were changing, identical experiments separated in time by several days were examined, and the number of intervening samples was determined from the data records. The number of field reversals per sample was similarly estimated.

\section{RESULTS}

Results for $40 \mathrm{mM}$ erbium content are shown in Figure 1, where the upper curve is from the first recorded experiment using an effective concentration of erbium. (About 45 samples were run in the process of setting up the HGMS system, but unfortunately data for these were not recorded; consequently, the performance of the matrix when first installed is unknown.) The central curve came after 52 intervening sample runs representing about 420 field reversals at various field intensities. The lower curve represents results for a sample some 59 runs (and 470 field reversals) after that of the central curve. Differences are insignificant for magnetization fields of 0.5 Tesla or greater; in contrast, filtration efficiencies progressively decreased for magnetizations less than this intensity.

Because there was no reason to expect matrix characteristics to stabilize, experiments using the $316 \mathrm{~L}$ wire were discontinued and the matrix was replaced with mesh woven of 430 ferritic wire 50 micra in diameter, packed to $15.5 \%$ of chamber volume. Comparative data for this matrix are indicated in the figure by unconnected " $x$ " symbols. Since all other HGMS and cell parameters are constant, an estimate of the effective magnetization attained by the 316L matrix can be obtained through use of the filter equation developed by Watson ${ }^{7}$, $(1-E)=\exp (-B)$, where $E$ is the magnetic filtration efficiency in percent divided by 100 and $\beta$ is proportional to both the saturation magnetization and packing factor of the wire, but is inversely proportional to the square of its radius. 


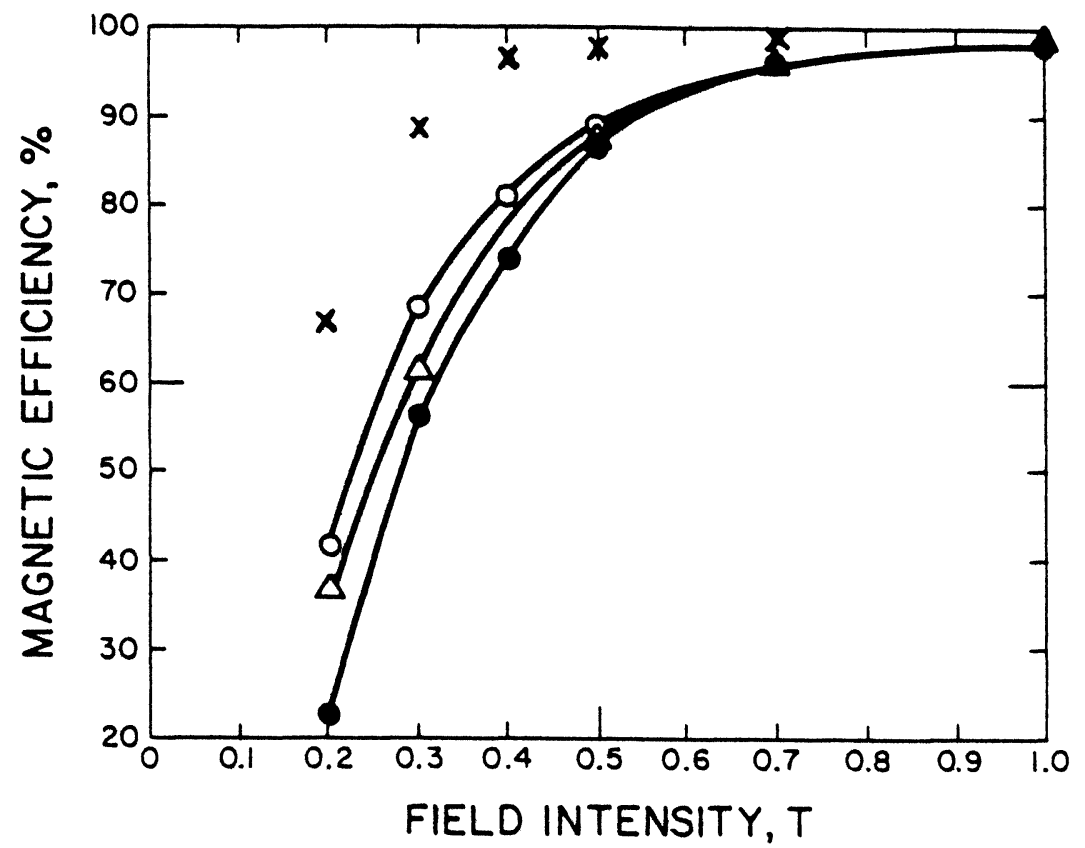

FIGURE 1 Degradation seen in filtration efficiency when $316 \mathrm{~L}$ stainless wire was used as matrix in blood-cell filtration. Open circles indicate results of first recorded filtration, open triangles the 53rd, and filled-in circles the 112 th. Comparative data $(x)$ are also included for 430 stainless wire.

For a magnetization field of 0.3 Tesla, filtration efficiencies of 68,61 , and 56 percent were obtained in the three experiments, in contrast to 87 percent for the ferritic matrix both when installed and after a comparable number of filtration runs. When Watson's equation is applied to these data points, estimates of 37,31 , and 27 percent of the ferritic saturation magnetization result for the $316 \mathrm{~L}$ matrix. Decreases in effective saturation magnetization of 17 and 28 percent, compared to the earliest curve, are apparent in later ones.

\section{DISCUSSION AND CONCLUSION}

Austenitic stainless alloys are often regarded as being non-magnetic. However, when cold worked as in drawing into fine wires, several exhibit considerable ferromagnetism ${ }^{8}$. Thought to originate in fine carbide precipitates and quasi-martensitic ferrite ${ }^{8}$, magnetic properties of commercial austenitic alloys can be explained through a two-phase model. Drawing tends to align both austenitic and ferritic 
phases in the direction of drawing, the perfection of alignment increasing with repeated drawing operations. For unannealed wires a few micra in diameter, alignment of the ferritic phase may be essentially perfect across a large percentage of the diameter, and saturation magnetizations can approach those of ferritic stainlesses. Grain alignment and magnetic properties are both lost on annealing.

As demonstrated here, magnetic properties can also be degraded through non-thermal processes. While the mechanism is not well understood, it is likely to involve intergranular corrosion resulting from the combination of chloride-containing reagents and demagnetizing field reversals. Stainless steels derive their superior corrosion resistance from a thin protective layer of reaction products which forms on their exposed surfaces ${ }^{10}$; these are subject to first pitting, then crevice, attacks on non-metallic sulphide inclusions at these surfaces ${ }^{11}$. Chloride ions are a particularly aggressive agent of corrosion11,12. Mechanical abrasion and fretting permit alternate avenues of corrosive attack, through removal of the passivating layer, and fretting could be observed by microscopy on surfaces of the $316 \mathrm{~L}$ wire when it was removed from the filter chamber. Also visible was increased surface roughness, tending to be deeper along the axis of the wire; this lends credence to the probability that corrosional losses of intergranular precipitates are involved in the degrading mechanism. Although corrosional processes are usually diffusion limited, there is some evidence that magnetic fields may augment corrosion rates, by magnetohydrodynamically removing corrosion products ${ }^{3}$; this process would be favored by the field reversals.

As a matrix material, 316L alloy should be restricted to applications where field reversals are seldom required, particularly if it is exposed to corroding fluids such as saline. A similar precaution probably applies to the other austenitic alloys.

\section{REFERENCES}

1. C.S. Owen, Biophysical Journal, 22, 171 (1978).

2. C.S. Owen, et al, J. Immunology, 123, 1778 (1979).

3. C.S. Owen and N.L. Sykes, J. Immunolog. Methods, 73, 41 (1984).

4. M.D. Graham and P.R. Selvin, IEEE Trans. Magnetics, MAG-18, 1523 (1982).

5. M.D. Graham, J. Applied Physics, 52, 2578 (1981).

6. M.D. Graham, Annals NY Academy Sciences, 435, 545 (1984).

7. J.H.P. Watson, J. Applied Physics, 44, 4209 (1973).

8. P.T. Hobson, E.S. Chatt and W.P. Osmond, J. Iron \& Steel Institute, 159, 145 (June 1948).

9. E.W. Collings and S.C. Hart, Cyrogenics, 19, 521 (1979).

10. I. Olefjord, Materials Science \& Engineering, 42, 161 (1980).

11. T. Sydberger, Werkstoffe \& Korrosion, 32, 119 (1981).

12. B. -0. Elfst rom, Materials Science \& Engineering, 42, 173 (1980).

13. M.E. Ghabashy, G.H. Sedahmed and I.A.S. Mansour, British Corrosion Journal, 17, 36 (1982). 